Preoperative ultrasound compared to final pathology report showed a ICC of $0-87(0.8-0.91)$ for maximum diameter size and $0.64(0.4-0.78)$ for tumour volume measurement.

Conclusion Maximum diameter size showed a good correlation (ICC $=0.75-0.9)$ with the pathology report when measured preoperatively by ultrasound and a moderate correlation (ICC $=0.5-0.75)$ when measured by MRI. For tumour volume measurement both ultrasound and MRI showed a moderate correlation with the final pathology report.

Disclosures All authors contributed to writing the manuscript and read and approved the final manuscript.

The authors declare no conflict of interest.

\section{SHOULD WE REALLY ABANDON MINIMALLY INVASIVE SURGERY IN EARLY-STAGE CERVICAL CANCER? ONCOLOGICAL RESULTS OF LAPAROSCOPICALLY ASSISTED RADICAL VAGINAL HYSTERECTOMY}

${ }^{1}$ Ariel Gustavo Glickman, ${ }^{1}$ Jaume Pahisa, ${ }^{2}$ Blanca Gil Ibanez, ${ }^{1}$ Berta Diaz-Feijoo, ${ }^{1}$ Pere Fusté, ${ }^{3}$ Núria Carreras, ${ }^{3}$ Núria Agustí, ${ }^{3}$ Lydia Gaba, ${ }^{4}$ Marta Del Pino, ${ }^{1}$ Aureli Torne. ${ }^{1}$ Hospital Clínic Barcelona; Gynaecological Oncology Unit; ${ }^{2}$ Gynecological Oncology and Endoscopy Unit. Gynecology and Obstetrics Department. University Hospital 12 de Octubre. Madrid. Spain. Research Institute I+12. University Hospital 12 de Octubre. Madrid; ${ }^{3}$ Hospital Clínic Barcelona; ${ }^{4}$ Hospital Clínic; Hospital Clínic Barcelona; Gynaecology

\subsection{6/ijgc-2020-ESG0.35}

Introduction/Background Recent evidence indicates that some minimally invasive surgery (MIS) approaches, such as laparoscopic- and robotic-assisted radical hysterectomy, offer lower survival rates to patients with early-stage cervical cancer compared with open radical hysterectomy. We evaluated the oncological results of a different MIS approach, that of laparoscopically assisted radical vaginal hysterectomy (LARVH) in the treatment of patients with early-stage cervical cancer.

Methodology From January 2001 to December 2018, patients with early-stage cervical cancer (IA1 with lymphovascular invasion, IA2, IB1, and IIA $<2 \mathrm{~cm}$; FIGO 2009) were treated by LARVH. Colpotomy and initial closure of the vagina were performed following the Schauta procedure, avoiding manipulation of the tumor. Laparoscopic sentinel lymph node (SLN) biopsy was performed in all cases. Women treated between 2001 and 2011 also underwent systematic bilateral pelvic lymphadenectomy after SLN biopsy. Adjuvant radiotherapy or chemo-radiotherapy was administered according to standard guidelines.

Results One hundred fifteen patients were included. Intraoperative complications occurred in nine patients $(7.8 \%)$. Adjuvant radiotherapy or chemoradiotherapy was administered to $35(30.4 \%)$ and three $(2.6 \%)$ patients, respectively. After a median follow-up of 87.8 months (range 1-216), seven women $(6 \%)$ presented recurrence (three pelvic and two paraaortic recurrences, and two had distant metastases). Four women died (mortality rate 3.4\%). The three and 4.5year disease-free survival rates were $96.7 \%$ and $93.5 \%$, respectively, and the overall survival was $97.8 \%$ and $94.8 \%$, respectively.

Conclusion LARVH offers excellent disease-free and overall survival in women with early stage cervical cancer and can be considered as an adequate MIS alternative to open radical hysterectomy.

Disclosures No disclosures to declare.

\section{5 IMPACT OF AGE ON CANCER SPECIFIC SURVIVAL IN PATIENTS WITH LOCALLY ADVANCED CERVICAL CANCER}

${ }^{1}$ David Cantu-de Leon, ${ }^{2}$ Lenny Gallardo-Alvarado, ${ }^{2}$ Rebeca Ramirez-Morales, ${ }^{3}$ Salim Barquet-Muñoz, $\quad{ }^{3}$ Rosa Salcedo-Hernandez, ${ }^{4}$ Gabriel Santiago-Concha, ${ }^{4}$ Sandra Perez-Alvarez, ${ }^{5}$ Delia Perez-Montiel, ${ }^{2}$ Paulina Trejo-Guerra. ${ }^{1}$ Insituto Nacional de Cancerología; Research; ${ }^{2}$ Instituto Nacional de Cancerología; Clinical Research; ${ }^{3}$ Insituto Nacional de Cancerología; Gynaecology; ${ }^{4}$ Instituto Nacional de Cancerología; Radiotherapy; 5 Instituto Nacional de Cancerología; Pathology

\subsection{6/ijgc-2020-ESG0.36}

Introduction/Background Cervical Cancer (CC) is uncommon in very young $(<35$ years $)$ and in geriatric women $(>65$ years), age as a prognostic factor is still controversial. The extremes of life had certain risk factors for being diagnosed with locally advanced cervical cancer (LACC); one of them is that in young women, there is a belief that the disease does not occur; therefore, lack of knowledge of the signs, symptoms and, as an essential factor, a lack of adherence to screening is common. In women older 65 years, the screening has been suspended, explaining how this group of women tend to be diagnosed in advanced stages.

This work aims to compare sociodemographic, clinical, and pathological characteristics, response to treatment, disease-free survival, overall survival, and cancer-specific survival in patients with LACC treated with concurrent chemoradiotherapy, clustered by age.

Methodology It is a retrospective study in patients with LACC treated at the National Cancer Institute of Mexico City from 2005 to 2014. A descriptive, comparative, and survival and cancer specific analysis was conducted.

Results From a total of 2,091 patients with LACC, we found 125 patients (9.7\%) younger than 35 years (group 1), 533 (41.35), age between 36-50 years (group 2), 444 (34.4\%) between 51-65 years (group 3) and $189(14.6 \%)$ of patients 66 years or older. The general characteristics are found in table 1 . More than $50 \%$ of women from group 4

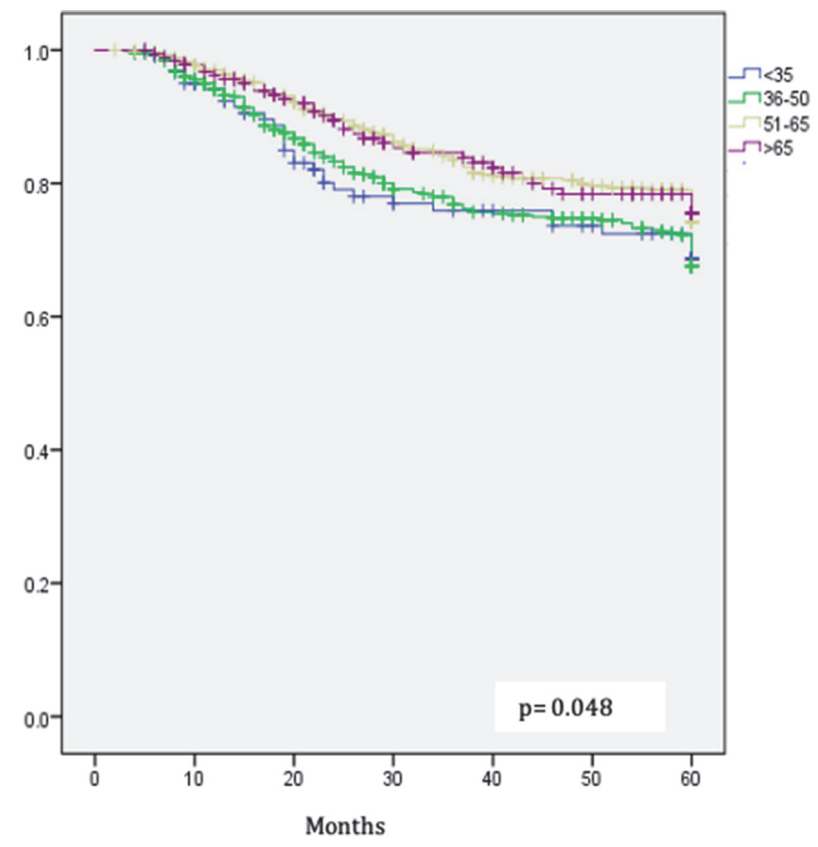

Abstract 445 Figure 1 Cancer specific survival 
Abstract 445 Table 1 Demographic, clinical and pathological characteristics

\begin{tabular}{|c|c|c|c|c|c|}
\hline & $\begin{array}{l}\text { Grupo } 1 \\
n=125\end{array}$ & $\begin{array}{l}\text { Grupo } 2 \\
n=533\end{array}$ & $\begin{array}{l}\text { Grupo } 3 \\
n=444\end{array}$ & $\begin{array}{l}\text { Grupe } 4 \\
n=189\end{array}$ & $\boldsymbol{P}$ \\
\hline Scholarship & & & & & 0.001 \\
\hline Illiterate & $5(4.0)$ & $48(9.0)$ & $129(29.1)$ & $97 \quad(51.3)$ & \\
\hline$>12$ years of edasation & $4(3.2)$ & $13(2.4)$ & $5(1.1)$ & 0 & \\
\hline Clinical Stage & & & & & 0.001 \\
\hline IB2 & $24(19.2)$ & $51(9.6)$ & $23(5.2)$ & $9(4.8)$ & \\
\hline III & $6(4.8)$ & $22(4.1)$ & $24(5.4)$ & $14(7.4)$ & \\
\hline IIB & $61(48.8)$ & $300(56.3)$ & $271(61.0)$ & $112(59.3)$ & \\
\hline III & $31(24.8)$ & $148(27.8)$ & 115 (25.9) & $52(27.5)$ & \\
\hline IVA & $3(2.4)$ & $12(2.3)$ & $11(2.5)$ & $2(1.1)$ & \\
\hline Histology & & & & & 0.005 \\
\hline Squamous cell carcinoma & $114(91.2)$ & $462(86.7)$ & $397(89.4)$ & $191(95.8)$ & \\
\hline Adenocarcinoma & $11(8.8)$ & $71(13.3)$ & $47(10.6)$ & $8(4.2)$ & \\
\hline Grade & & & & & 0.534 \\
\hline 1 (well differentiated) & $3(2.4)$ & $23(4.3)$ & $12(2.7)$ & $3(1.6)$ & \\
\hline 2 (moderately differentiated) & $94(75.2)$ & $390(73.2)$ & $327(73.6)$ & $137(725)$ & \\
\hline 3(poorlyilundiffereatizted) & $28(22.4)$ & $120(225)$ & $105(2.3 .6)$ & $49(25.9)$ & \\
\hline Lymphovascular space invasion & $13(10.4)$ & $48(9.0)$ & $57812.8)$ & $16(8.5)$ & 0.194 \\
\hline Tumour Size $4 \mathrm{~cm}$ & $99(79.2)$ & $409(67 . \pi)$ & $274(61.4)$ & $110(58.2)$ & $<0.001$ \\
\hline Parametrial involvement & & & & & 0.18 \\
\hline Negative & $30(24.0)$ & $74(13.9)$ & $49(11.0)$ & $24(12 \pi)$ & \\
\hline Positive but not up to the pelvic wall & $\cos (52.8)$ & $327(61.4)$ & $291(65.5)$ & $127(67.2)$ & \\
\hline Extension to the pelvic wall & $29(23.2)$ & $132(24.8)$ & $104(23.4)$ & $38(20.1)$ & \\
\hline Pelvik Lymph nodes & $50(40)$ & $201(37.7)$ & $148(24.3)$ & $46(24.3)$ & 0.014 \\
\hline Pre-treatment Hacmoglobin & $12.3(5.3-16.1)$ & $10.6(3.5-21.1)$ & $13.8(5.6-20.3)$ & $13.7(7.1-16.7)$ & 0.002 \\
\hline Final hacmoglobin & $12.1(6.7 .15 .1)$ & $12.2(5.5-16.1)$ & $12688.4 \cdot 15.9)$ & $12.4(6.3-13.4)$ & 0.288 \\
\hline Blood transfusion & $59(58.4)$ & $175(39.8)$ & $92(24.7)$ & $31(18.9)$ & $<0.001$ \\
\hline Treatment outcome & $n(\%)$ & $n(\%)$ & $n(\%)$ & $\mathrm{n}(\%)$ & $<0.001$ \\
\hline Complete respoess & $94(75.2)$ & $406(76.2)$ & $390(85.6)$ & $108(87.8)$ & \\
\hline Partial, progression of stable disease & $26(20.8)$ & $119(223)$ & $27(12.8)$ & $19(10.1)$ & \\
\hline Unknown & $12(4.5)$ & $8(1.5)$ & $7(1.6)$ & $4(2.1)$ & \\
\hline Recurrence & $28(22.4)$ & $125(23.5)$ & $119(26.8)$ & $6(31.7)$ & 0.111 \\
\hline Type of recurrence & & & & & 0,512 \\
\hline Local & $2(1.6)$ & $17(3.2)$ & $17(3.8)$ & $10(5.3)$ & \\
\hline Locoregional & $4(3.2)$ & $11(2.1)$ & $13(2.9)$ & $5(2.6)$ & \\
\hline Distance & $22(17.6)$ & $93(18.2)$ & $88(19.8)$ & $45(23.8)$ & \\
\hline Disease free survival (moaths) media (C195\%) & $48(44-52)$ & $48(46-50)$ & $48(46-50)$ & $45(42-48)$ & 0.263 \\
\hline Cancer Speeifle survival (moaths) media (C195\%) & $49(45-52)$ & $49(48-51)$ & $25(51-54)$ & $52(50-54)$ & 0.048 \\
\hline Overall survival (months) medin (C195\%) & $48(44-51)$ & $49(50-53)$ & $52(50-53)$ & $51(49.54)$ & 0.006 \\
\hline
\end{tabular}

were illiterate. The patients from groups 1 and 2 clinical stage IB2 was more common. Pre-treatment haemoglobin was lower in groups 1 and 2 vs. groups 3 and 4, and $58.4 \%$ of the patients in group 1 required at least one blood transfusion. Cancer-specific survival was different between groups 1 and 2 vs. 3 and 4, p=0.048 (figure 1). Multivariate analysis showed that clinical-stage, Hazard ratio (HR) 3.62 (CI 95\% 1.59-8.20), pre-treatment haemoglobin HR 0.944 ( CI 95\% 0.89-0.99), and age HR 1.28( CI 95\% 1.02-1.64) are independent prognostic factors in patients with LACC, with lack of significance in disease free survival and overall survival.

Conclusion There are demographic, clinical, and treatment response differences between very young and young patients (under 50 years) compared to older patients (over 50 years). Cancer-specific survival, which attempts to remove the bias of advanced age in mortality, showed that women younger than 50 years had higher cancer-related mortality than those of older ages.

Disclosures The authors reports no conflicts of interest. 